\title{
Grund und Grenzen der Nothilfe
}

\author{
Christian Jäger*
}

Rezension zu Armin Engländer, Grund und Grenzen der Nothilfe, Tübingen (Mohr Siebeck) 2008. XII, 412 S., 94,00 €, ISBN 978-3-16-149723-O

Jeder Versuch, die weit über die Dogmatik des Notwehr- und Nothilferechts hinausgehende Konzeption Engländers vollständig nachzuzeichnen, wäre im Rahmen einer Rezension von vornherein zum Scheitern verurteilt. Seine methodisch subtile und in der Sache konzentrierte Argumentation kann daher nur um den Preis des Verzichts auf Substanz und Genauigkeit zu einem Überblick kondensiert werden.

Schon in der Einleitung (S. 1 bis 6) steckt Engländer in sechs Punkten das Programm ab, das er sodann auf knapp 400 Seiten abarbeitet. Grundlage dieses Programms ist der Legitimationsgedanke der Nothilfe und die damit zusammenhängende Frage, ob der Nothelfer lediglich stellvertretend die Verteidigungsbefugnis des Angegriffenen wahrnimmt oder aus eigenem Recht handelt.

\section{A. Der Legitimationsgrund der Nothilferechtfertigung}

In einem ersten Schritt untersucht Engländer zunächst die ratio legis der Nothilfe (S. 7 bis 98), die er in der Folge konsequent zur Basis seiner weiteren Überlegungen macht. Er kommt dabei zu dem Ergebnis, dass Notwehr und Nothilfe allein dem Schutz der Individualgüter des Angegriffenen und nicht ausschließlich oder zusätzlich der Bewahrung der Rechtsordnung zu dienen bestimmt sind. Dementsprechend lehnt er nicht nur das überindividualistische, sondern auch die dualistischen Notwehr- und Nothilfemodelle ab. Das überindividualistische Modell, so Engländer, verstoße gegen den Wortlaut des $\$ 32$ Abs. 2 StGB, der mit der Wendung „von sich oder einem anderen “ den Angriff auf ein Individualrechtsgut verlange. Aus dem gleichen Grunde sei auch dasjenige dualistische Modell abzulehnen, das alternativ entweder das Vorliegen eines Individualschutzinteresses oder eines Rechtsbewährungsinteresses verlange. Denn soweit nur letzteres vorliege, verbiete wiederum der Wortlaut die Zubilligung eines Notwehr- oder Nothilferechts. Aber auch das dualistische Modell in seiner kumulativen Form lehnt Engländer ab. Denn wer für Notwehr und Nothilfe neben dem Individualschutz auch ein Rechtsbewährungselement fordere, der müsse bei vorhandenem Individualgüterschutzinteresse und fehlendem Rechtsbewährungsinteresse ein Notwehr- und Nothilferecht verneinen, obwohl $\mathbb{} 32$ Abs. 2 StGB nur verlange, dass die Verteidigung erforderlich sein muss, um den Angriff von sich oder einem anderen abzuwenden (S. 31). Seinem individualistischen Standpunkt (S. 38 ff.) entsprechend weist Engländer in diesem Zusammenhang kon-

* Prof. Dr. Christian Jäger lehrt Strafrecht und Strafprozessrecht, insbesondere Wirtschaftsstrafrecht und Medizinrecht an der Universität Bayreuth. 
sequent nach, dass der Nothelfer nicht aus eigenem Recht handele, sondern die aus der Schutzfunktion der subjektiven Rechte des Angegriffenen resultierende Verteidigungsbefugnis ( $\mathrm{S} .67 \mathrm{ff}$.) lediglich stellvertretend für diesen wahrnehme, so dass von einem akzessorischen Charakter der Nothilfe auszugehen sei (S. 90 f.).

\section{B. Die Akzessorietät der Nothilfe}

In einem zweiten Schritt wendet sich Engländer diesem Gesichtspunkt der Akzessorietät näher zu und untersucht die Frage nach der Bedeutung des entgegenstehenden Willens des Angegriffenen und das damit zusammenhängende Problem der Willensermittlung (S. 99 ff.). Engländer unterscheidet dabei im Anschluss an Seier ${ }^{1}$ in interessanter Weise zwischen bloßem Beistandsverzicht einerseits und echtem Nothilfeverbot andererseits (S. 102). Nur in letzterem Fall, so Engländer, lehne der Angegriffene die Verteidigung durch einen anderen tatsächlich ab. Von einem Verzicht sei dabei regelmäßig nur dann auszugehen, wenn es dem Angegriffenen um die Schonung des Nothelfers gehe. Im Übrigen sei die Ablehnung - entgegen Seier - grundsätzlich als Verteidigungsverbot aufzufassen.

Was die Erklärung des Willens anbelange, so sei der ausdrücklich oder konkludent geäußerte Wille maßgeblich; sei ein solcher nicht feststellbar, so komme es auf den mutmaßlichen Willen an. Diesem Ausgangspunkt entsprechend untersucht Engländer im Hinblick auf die Beachtlichkeit des Verteidigungsverbots die aus der Einwilligungsdogmatik bekannten personenbezogenen, rechtsgutsbezogenen und willensmängelbezogenen Unwirksamkeitsgründe. Hierbei macht er gravierende Unterschiede im Verhältnis zur Einwilligungslehre aus. So seien etwa Kollektivinteressen, die für Einwilligungsschranken bei Eingriffen in Leib und Leben angeführt werden, auf den die Nothilfe betreffenden Ablehnungswillen nicht übertragbar (S. 138 f.). Engländer sieht hier den maßgeblichen Unterschied darin, dass es bei der Ablehnung der Nothilfe, anders als bei der Einwilligung, nicht darum gehe, den Eingriff zu gestatten (die Tat bleibt eine rechtswidrige), sondern nur darum, das Recht auf Gegenwehr nicht in Anspruch nehmen zu wollen. Demzufolge könne der ausschlaggebende Grund für die Unbeachtlichkeit des Ablehnungswillens bei Eingriffen in das Leben nur in einem Übereilungsschutz gesehen werden.

Auch bei irrtumsbedingten oder zwangsbedingten Willensmängeln weist Engländer auf die Unterschiede zwischen Einwilligung und Nothilfeverzicht hin: Im Fall der Einwilligung bedeute die irrtums- bzw. zwangsbedingte Unwirksamkeit eine Handlungsbeschränkung des Akteurs, während eine aus den gleichen Gründen zu verzeichnende Unwirksamkeit der Nothilfeablehnung eine Handlungserweiterung zur Folge habe. Es spreche daher alles dafür, Irrtümer unabhängig davon, ob sie rechtsgutsbezogener Natur sind, für beachtlich zu erklären und eine Unwirksamkeit des

1 J. Seier, Umfang und Grenzen der Nothilfe im Strafrecht, NJW 1987, 2476 (2482 f.). 
zum Ausdruck gebrachten Ablehnungswillens anzunehmen (S. 142 f.). Ausschlaggebend sei daher in derartigen Fällen allein der mutmaßliche Wille des Angegriffenen. Gleiches gelte bei zwangsbedingten Willensmängeln. Denn auch hier besage eine unmittelbar erzwungene Ablehnungserklärung prinzipiell nichts über die tatsächliche Erwünschtheit oder Unerwünschtheit der Nothilfe, so dass wiederum der mutmaßliche Wille des Angegriffenen zum Tragen komme (S. 145 mit Beispielen). Was sodann motivbezogene Ablehnungsgründe anbelangt, so kommt Engländer seinem individual-akzessorischen Ansatz entsprechend zu dem Ergebnis, dass diese Beweggründe grundsätzlich zu respektieren seien und zu einem Ausschluss der Verteidigungsbefugnis durch den Dritten führten (S. 146 ff.). Dagegen sei für die Zulässigkeit der Nothilfe zugunsten eines sie billigenden Angegriffenen der Ablehnungswille eines anderen gleichzeitig Angegriffenen unbeachtlich (S. 150 f.).

\section{Das Verhältnis von privater Nothilfe und staatlicher Gefahrenabwehr}

I. Hinsichtlich des Konkurrenzverhältnisses zwischen privaten und staatlichen Abwehrmaßnahmen spricht sich Engländer im dritten Kapitel klar für einen grundsätzlichen Vorrang möglicher staatlicher Gefahrenabwehr aus. Der Nothelfer müsse sich daher bei Erreichbarkeit staatlicher Hilfe prinzipiell zurücknehmen. Ausnahmen will Engländer nur für den Fall anerkennen, dass der Hoheitsträger zwar präsent ist, aber nicht handeln darf, nicht handeln will oder lediglich eine Maßnahme ergreift, die ein höheres Fehlschlagsrisiko birgt als die private Nothilfe. Deshalb hält Engländer auch ein Herbeiholen hoheitlicher Hilfe nur dann für erforderlich, wenn dies ohne erhöhte Risiken für den Angegriffenen möglich sei (vgl. zum Ganzen S. 159 ff.).

Dieser Standpunkt führt zu bemerkenswerten Konsequenzen, sofern man - mit Engländer (vgl. S. 332 ff., dazu unter F.) - die Folter durch Private für grundsätzlich zulässig erklärt: Gibt der Entführer eines Kindes das Erdloch, in dem er das Opfer versteckt hat, nicht preis, so könnte der Vater des Kindes die Aussage durch Folterung des Entführers erlangen, selbst wenn Polizeibeamte zugegen sind und diesen zur Folter keine Befugnis zusteht. Die Polizeibeamten dürften in diesem Fall also nicht einschreiten, weil das Nothilferecht des Vaters trotz der Präsenz von Polizeikräften erhalten bliebe.

II. Was sodann das Verhältnis von Nothilfe und staatlicher Abwehr in Bezug auf den handelnden Hoheitsträger selbst anbelangt, so tritt Engländer für die sog. Aufspaltungslösung ein: Das Handeln des Hoheitsträgers richte sich ausschließlich nach öffentlichem Recht, so dass die Notwehr- und Nothilferegelungen nicht in der Lage seien, Eingriffsbefugnisse zu verleihen. Sie könnten nach dem Konzept Engländers vielmehr nur das Strafunrecht der Tat entfallen lassen und so auf der Ebene der 
Sanktionsnormen im Anschluss an Günther ${ }^{2}$ als Strafunrechtsausschließungsgrund wirksam werden (S. $216 \mathrm{ff}$.$) .$

\section{Die Nothilfelage}

Interessant sind auch Engländers Überlegungen zur Rechtswidrigkeit des Angriffs im Rahmen der Nothilfelage (S. 234 ff., 256 ff.). Engländer legt hier wiederum seinen streng subjektiv-rechtlichen Ansatz zugrunde, wonach die Ratio von Notwehr und Nothilfe einen Verstoß gegen subjektive Rechte eines Rechtsträgers erfordere. Engländer folgert hieraus, dass nur derjenige rechtswidrig angreifen könne, der überhaupt Adressat dieser Rechte sei. Mangels Einsichts- und Steuerungsfähigkeit sei dies bei Kindern und Geisteskranken nicht der Fall, so dass es bei ihnen grundsätzlich an einem rechtswidrigen Angriff fehle. Notwehr und Nothilfe seien daher gegen sie unzulässig. Es bleibt dann, so muss ergänzt werden, nur eine mögliche Rechtfertigung nach $\mathbb{} 34$ StGB. Dagegen verfügten Betrunkene und unter Drogeneinfluss stehende Angreifer zumindest prinzipiell über Einsichts- und Steuerungsvermögen, so dass sie Adressaten der subjektiven Rechte anderer blieben. Ihr Angriff bleibe daher ein rechtswidriger. Dies gelte umso mehr, als diese auch dem Verlust dieser Fähigkeiten entgegenwirken könnten.

\section{E. Die Nothilfehandlung}

Auch hier arbeitet Engländer mit Blick auf das Merkmal der Erforderlichkeit (vgl. S. 277 ff.) bedeutungsvolle Abhängigkeiten zwischen potentieller Selbstverteidigung und möglicher Nothilfe heraus: Danach darf der Nothelfer kein eingriffsintensiveres Mittel verwenden als dasjenige, das dem Angegriffenen selbst genügt hätte (S. 284 ff.). Umgekehrt darf der Angegriffene, der sich nicht von einem Nothelfer verteidigen lassen will, nicht zu stärkeren Mitteln greifen, als letzterer zur sicheren Abwehr benötigt hätte (S. 289 ff.).

\section{F. Die Nothilfeeinschränkungen}

Im letzten Abschnitt seines Buches wendet sich Engländer schließlich dem Problem der Nothilfeeinschränkungen zu. Engländer hält diesbezüglich eine Herleitung aus dem Gebotenheitsmerkmal mangels Bestimmtheit dieses Kriteriums für ausgeschlossen und benennt stattdessen am Schutzzweck orientierte norminterne sowie normexterne Beschränkungsgründe. Interne Schranken leitet er aus der Akzessorietät der Nothilfe und aus dem Gedanken der missbräuchlichen Rechtsausübung - etwa bei der Absichtsprovokation - ab (S. 315 f). Externe, d. h. aus anderen Normen fließende Grenzen findet er dagegen im Solidaritätsprinzip, das in Fällen eines extremen Missverhältnisses zwischen Eingriffs- und Erhaltungsgut sowie in Situationen sonst vorwerfbarer Provokationen zum Tragen komme (S. 331 ff.). Sonstigen Einschränkun-

2 H.-L. Günther, Strafrechtswidrigkeit und Strafunrechtsausschluß, Köln et al. 1983, S. 253 ff. 
gen des Notwehrrechts (etwa aufgrund familiärer Beziehungen, S. 358 ff.) steht Engländer dagegen grundsätzlich ablehnend gegenüber. Insbesondere verneint er eine Erstreckung des Verbots der Rettungsfolter auf Private (S. 333 ff.)

\section{G. Abschließende Bemerkungen}

Zum reichen Gehalt dieses Buches im Einzelnen Stellung zu nehmen, hieße, eine unlösbare Aufgabe im Rahmen einer Rezension zu bewältigen.

Insgesamt steht und fällt Engländers Konzept mit seiner individualrechtlichen Deutung des Notwehr- und Nothilferechts sowie der damit einhergehenden Ablehnung kollektiver oder dualistischer Auffassungen. Wie ein roter Faden zieht sich dieser Standpunkt durch das gesamte Werk. Die Schrift besticht hier nicht nur durch die Entschiedenheit und Eindeutigkeit ihrer Thesen, sondern auch durch die Kraft der klaren Argumentation. Dennoch wird man die Frage stellen müssen, ob das Notwehr- und Nothilferecht tatsächlich ganz unabhängig vom Prinzip der Rechtsbewährung bestehen kann. Denn wenn auch Engländer etwa bei der Absichtsprovokation zur Einschränkung des Notwehrrechts den Gedanken des Rechtsmissbrauchs bemüht, so ist dies doch ein Gesichtspunkt, der für die Bedeutsamkeit eines über das Individualgut hinausgehenden Rechtsbewährungsinteresses spricht.

Auch lassen sich bestimmte Folgerungen aus Engländers subjektiv-rechtlichem Ansatz durchaus bezweifeln. So scheint mir bestreitbar, dass etwa der betrunkene Angreifer rechtswidrig handelt, da er prinzipiell Adressat subjektiver Rechte sein kann, während dies bei Kindern und Geisteskranken nicht gelten soll. Denn wenn man die Rechtswidrigkeit allein auf die Tat bezieht und die Schuld auf den Täter, so ist mit dem Kriterium (potentieller) normativer Ansprechbarkeit doch eher der Gesichtspunkt personaler Verantwortlichkeit verbunden, wie auch $\$ 17$ StGB belegt.

Ebenso wird man bestreiten dürfen, dass etwa der Private, der zur Rettungsfolter schreitet, selbst dann noch gerechtfertigt sein soll, wenn (nicht zur Folter befugte) Polizisten anwesend sind. Denn Folge dieser Auffassung wäre, dass die Polizei dazu verurteilt wäre, den möglicherweise grausamen Folterungen des Privaten zuzusehen oder die Augen vor ihnen zu verschließen. Mit der Würde des Rechtsstaates wäre dies aber kaum vereinbar. ${ }^{3}$

Lässt man jedoch Einzelheiten dieser Art beiseite, so ist generell festzustellen, dass sich die Ausführungen Engländers durch äußerst behutsames Abwägen, durch gründliche Berücksichtigung des Für und Wider sowie durch eindringliche Stellungnahmen zu sämtlichen Streitfragen auszeichnen.

3 Vgl. zu diesem Aspekt bereits C. Jäger, Das Verbot der Folter als Ausdruck der Würde des Staates, in: H. Putzke et al. (Hrsg.), Strafrecht zwischen System und Telos - Festschrift für Rolf Dietrich Herzberg, Tübingen 2008, S. 539 (544 ff.), ders., Folter und Flugzeugabschuss - rechtsstaatliche Tabubrüche oder rechtsguterhaltende Notwendigkeiten?, JA 2008, 678 (680f.). 
Darüber hinaus beansprucht das Thema der Nothilfe gerade im Hinblick auf das Problem der Rettungsfolter höchste Aktualität. Die Fragen, die mit dieser Problematik verbunden sind, harren nach wie vor einer endgültigen Lösung und sind weiterhin Gegenstand heftiger Auseinandersetzung. Hier erfüllt das Werk Engländers eine bedeutsame Aufgabe. Indem es mit Unbestechlichkeit und Sachlichkeit wissenschaftlicher Methodik die Probleme zu klären unternimmt, entrückt es sie dem politischen Streit und hebt es auf jene Ebene, die ihnen in einem Rechtsstaat allein angemessen ist: die Ebene des Rechts und der Rechtswissenschaft. Dabei wendet sich Engländer der bisher eher vernachlässigten Frage zu, ob das Verbot der Rettungsfolter auch für Private, etwa für den Vater eines Entführungsopfers gelten kann. Engländer verneint dies mit guten Gründen, auch wenn hier im Detail sicherlich noch weiterer Diskussionsbedarf besteht.

Alles in allem ist die Darstellung durch ihren durchdachten und umfassenden Charakter auch für rechtsphilosophische Überlegungen ${ }^{4}$ und für die wissenschaftliche Durchdringung des Notwehr- und Nothilferechts von großem Nutzen. Man wird daher bekennen müssen, dass die Schrift, gerade wenn man die Spärlichkeit der bisherigen Darstellungen zum Recht der Nothilfe betrachtet, einem echten Bedürfnis entspricht. Der Verfasser darf daher das Verdienst für sich in Anspruch nehmen, mit diesem Werk nicht nur eine große Lücke im strafrechtlichen Schrifttum geschlossen, sondern auch der künftigen Diskussion eine sichere Grundlage gegeben zu haben.

4 Erwähnen möchte ich hier nur Engländers Überlegungen zum Reziprozitätszusammenhang bei der Fremdschädigung und seinem Fehlen bei der Selbstschädigung, vgl. S. 130 f. Sie allein würden schon den Stoff für eine weitere Monographie hergeben. 